\title{
Poster Session
}

\section{LA.P10}

\section{A proton wire water channel revealed in the crystal structure of isatin hydrolase}

\author{
K. Bjerregaard-Andersen ${ }^{1}$, T. Sommer $^{1}$, J. Jensen ${ }^{3}$, B. Jochimsen ${ }^{3}$, M. Etzerodt ${ }^{3}$, J. Morth ${ }^{1,3}$ \\ ${ }^{1}$ Norwegian Center of Molecular Medicine, Nordic EMBL Partnership University of Oslo, Oslo, Norway, ${ }^{2}$ Institute for Experimental Medical Research, \\ Oslo University Hospital, Oslo, Norway, ${ }^{3}$ Department for Molecular Biology and Genetics, Aarhus University, Aarhus, Denmark
}

Two high resolution crystal structures of isatin hydrolase $(\mathrm{IH})$ from the Baltic seabed bacteria Labrenzia aggregata are presented. The crystals structure capture both the apo and the product state. This hydrolase present a new fold and the first metal-dependent hydrolase with this fold to be functionally characterized[1]. The Isatin hydrolase catalyze the reaction that convert isatin to isatinate and belongs to a novel family of metalloenzymes that include bacterial kynurenine formamidase (KynB) also recently published, however hoste a binclear zink site in the active site[2] as compared to a single manganese in $\mathrm{IH}$. The product state, mimicked by thioisatinate, has captured an additional water molecule that bridges the thioisatinate to a water channel and that could act as a proton wire and thus allows the proton to be released during the hydrolysis reaction only when the product is formed. The functional proton wire is therefore locked by thioisatinate and represents a unique catalytic feature trapped and visualized. Biochemical evidence for the proton wire is also presented as single point mutation from S225C enhances the Vmax of the enzyme. Ser-225 is the only side chain residue that is included in the proton wire. The molecular basis for thioisatinate recognition allows stronger and more confident identification of orthologous genes encoding isatin hydrolases within the prokaryotic kingdom. The isatin hydrolase orthologues found in human gut bacteria raise the question as to whether the indole-3-acetic acid degradation pathway is present in human gut flora.

[1] Bjerregaard-Andersen et al., J. Biol Chem 2014 June 10th (in press), [2] Diaz Saez L et al., Biochem J. 2014 Jun 19th (in press)

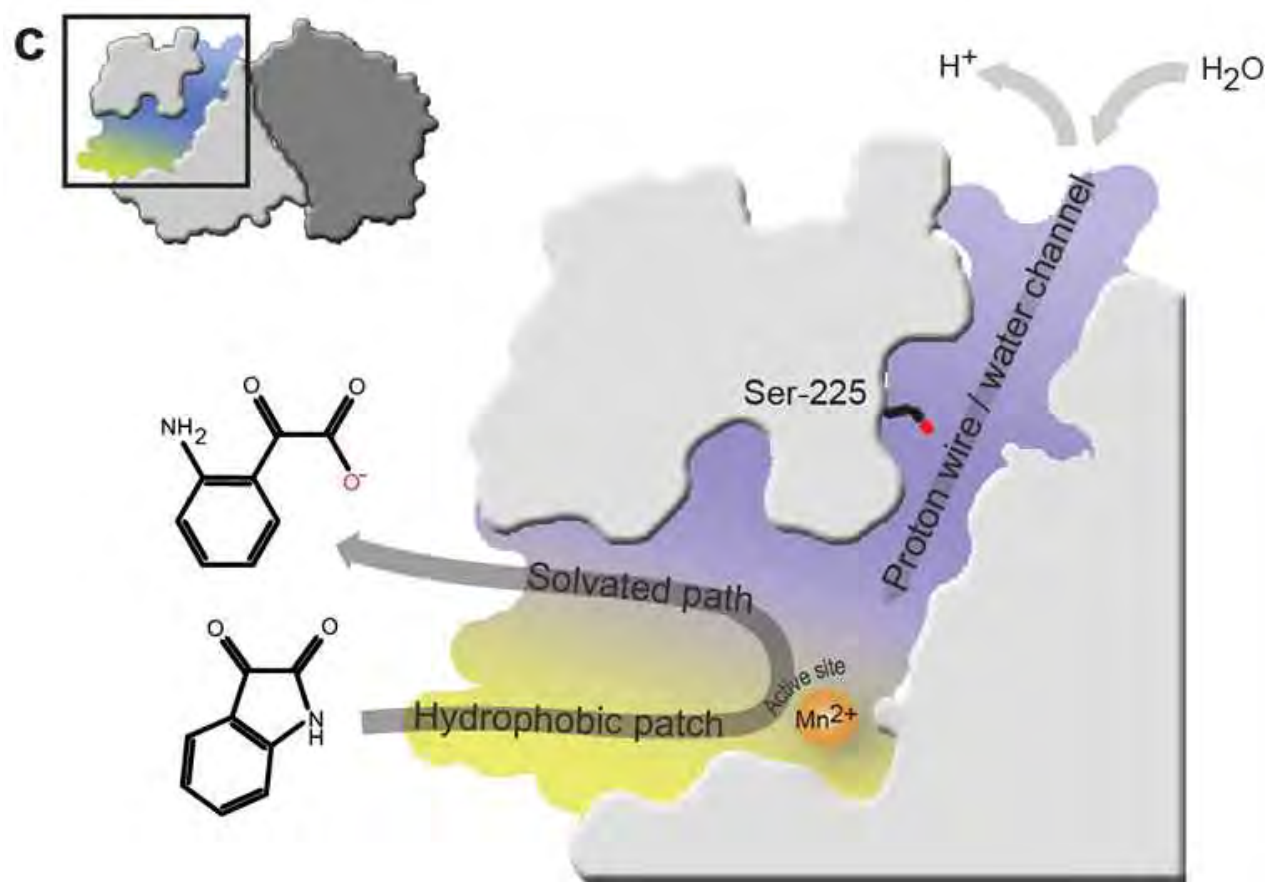

Keywords: Metalloenzyme, Isatin hydrolase, Grotthuss 